\section{Technische Unterstützung für Menschen mit Demenz?}

\author{
Zur Notwendigkeit einer \\ bedarfsorientierten Technikentwicklung
}

von Nora Weinberger und Michael Decker, Institut für Technikfolgenabschätzung und Systemanalyse (ITAS), Karlsruhe

Erste assistive Technologien für Menschen mit Demenz wurden bereits entwickelt und sind auch schon am Markt erhältlich. Von einem flächendeckenden Einsatz dieser Technologien kann aber nicht gesprochen werden. Vor dem Hintergrund der offensichtlich vorhandenen gesellschaftlichen Bedarfslage - Demenz gilt als eine der gesellschaftlichen Herausforderungen in Zusammenhang mit dem demografischen Wandel - liegt der Verdacht nahe, dass diese frühen technischen Entwicklungen die Nachfrage des Marktes noch nicht ausreichend gut adressieren. Hier kann eine bedarfsorientierte Technikentwicklung mit der Analyse des räumlichen, sozialen und funktionalen Handlungskontextes und der Identifikation der Anforderungen und Wünsche der Akteure im Pflegearrangement ansetzen. Das heißt, bevor technische Festlegungen getroffen werden, wird erhoben, welche Bedarfe an Unterstützung im Arrangement bestehen. Lassen sich zur Befriedigung dieser Bedarfe technische Assistenzsysteme identifizieren, die im Pflegearrangement für akzeptabel erachtet werden, dann beginnt ein anschließender Technikentwicklungsprozess bereits mit diesem Akzeptabilitäts-Vorschuss. Auch eine den Technikentwicklungsprozess begleitende Technikfolgenforschung profitiert von dieser bedarfsorientierten Vorgehensweise, wie anhand eines Fallbeispiels aus dem Pflegezusammenhang für Menschen mit Demenz dargestellt wird.

First assistive technologies for people with dementia have been developed and are on the market, yet a comprehensive use of these is still missing. Given the obvious existing social demand, one might suppose that these technological developments don't meet the market demand sufficiently so far. Here, a demand-oriented technology development with analysis of the spatial, social and functional contexts and an identification of the requirements and wishes of the various actors in care situations can help. This means that before concrete technologies are determined, an assessment of the needs of support in the care situations needs to take place. If technological assistance systems are developed based on these assessments that are regarded as acceptable, then the beginning technology development process already includes an advanced level of acceptability. Also, the technology development process accompanied by a technology assessment benefits from this demand-driven approach. This is demonstrated in a case study of a care situation of people with dementia.

\section{Gesellschaftliche Herausforderung „Demenz"}

Die Pflegesituation in Deutschland wird schon heute als prekär beschrieben (u. a. Geyer 2015). Das wird sich in den nächsten Jahren durch die steigende Anzahl von Menschen mit Demenz vermutlich noch verstärken. So wird die Zahl der Menschen, die weltweit mit Demenz leben auf 44 Mio. (bezogen auf 2014) geschätzt (Prince et al. 2014, S. 3). Ebenso wird erwartet, dass international die Zahl von Menschen mit Demenz von 65 Mio. im Jahr 2030 auf 115 Mio. im Jahr 2050 ansteigen wird (Ferri et al. 2005; Prince et al. 2014). Aktuell leiden in Deutschland 1,2 Mio. Menschen an einer leichten bis schweren Demenz.

Mit der Progredienz der Demenz geht ein zunehmender Verlust kognitiver Funktionen, einschließlich Gedächtnis, Denken, Lernfähigkeit, Sprache und Urteilsvermögen einher. Dieser zeigt sich u. a. in der abnehmenden Fähigkeit, Alltagsprobleme eigenständig zu lösen (Selbständigkeitsverlust). Ebenso nehmen Verkennungen, Angst und Wahn zu. Das Leben in Erinnerungen und die fortschreitende Orientierungslosigkeit in Raum und Zeit führen zu einer extremen Unruhe, die sich in einem hohen Bewegungsdrang (sog. "Wandering") äußern kann. Die Kombination von Orientierungslosigkeit und Bewegungsdrang kann zu einem Selbstgefährdungspotenzial führen, z. B. wenn der Weg zurück nicht mehr eigenständig gefunden werden kann oder Gefahren wie eine Autobahn bei einem Spaziergang nicht mehr als solche erkannt werden. Aufgrund der 
beschriebenen Ausprägungen beeinträchtigt Demenz allerdings nicht nur die Betroffenen selbst, sondern belastet in starkem Maße auch das Umfeld des Menschen mit Demenz. Hierdurch entsteht ein besonderer Bedarf an Unterstützung bei der Pflege von Menschen mit Demenz.

\section{Assistive Technologien als Lösung?}

Vor diesem Hintergrund der Veränderung der Altersstruktur der Gesellschaft im Allgemeinen und einem damit erwarteten Mangel an Pflegekräften ${ }^{1}$ rückt der Einsatz von assistiven Technologien ${ }^{2}$ in das Blickfeld. Solche Technologien scheinen das Potenzial zu haben, eine Lösung zur Abfederung der sich abzeichnenden Problemlage zu bieten (u. a. Haux et al. 2014). Diese Technologien sollen nach Beschreibung der Technikentwickler die Lebensqualität der Pflegebedürftigen verbessern sowie die Arbeit der Pflegekräfte erleichtern und damit die Effizienz und Effektivität von Pflegeleistungen erhöhen. Auch politisch werden technische Assistenzsysteme als ein Beitrag zur Lösung des Problems angesehen und ihre Einführung durch flächendeckende Beratungsdienstleistung unterstütz $\mathrm{t}^{3}$ (Mulvenna et al. 2010; Glende et al. 2011). Pflegevertretern ist die Relevanz von Technik in ihrem Pflegealltag ebenfalls bewusst, wie Hirsch ${ }^{4}$ herausarbeiten konnte. Exemplarisch sei hier ein Zitat einer Hausdirektion eines Pflegeheims aus dieser Arbeit genannt:

\begin{abstract}
„Und Technik kann unterstützen, Technik wird möglicherweise nicht die Pflege oder die menschliche Zuwendung ersetzen / nicht nur möglicherweise, sondern Hundertprozentig keine menschliche Zuwendung ersetzen können. Aber Technik kann helfen, dass Menschen mit ihren Beschränkungen weiterhin ein möglichst selbst bestimmtes und selbst verantwortetes Leben führen können.“
\end{abstract}

Viele assistive Systeme, die nach Aussage der Produktentwickler die beschriebenen Ansprüche adressieren, befinden sich aktuell in der Entwicklung oder sind als Prototyp in der Testphase. Darüber hinaus sind mehrere Hundert bereits am Markt verfügbar (vgl. u. a. Technikliste im Zukunftsradar Technik ${ }^{5}$ ). Doch trotz der Marktverfügbarkeit und der durch positive Evaluierung in Feldtests ausgewiesenen Potenziale wird bisher der Markt nicht durchdrungen, d. h., die Produkte kommen bis auf wenige Ausnahmen nicht im Pflegealltag an (u. a. Rachmann et al. 2011). Nun stellt sich die Frage, warum? Aus Sicht einiger Experten spielen hierbei verschiedene Aspekte eine Rolle: Zweifel an der Wirksamkeit (Roine et al. 2001; Hersh et al. 2001), die noch fehlende Kosteneffizienz (Whitten et al. 2002), die „Jugendlichkeit" des Pflege-Marktes, die geringen Bekanntheitsgrade der Technologien durch fehlende Multiplikatoren (Fachinger/Henke 2010), fehlende „Technik-Module“ in der Pflegeausbildung (Hülsken-Giesler 2010), keine oder ungeklärte Refinanzierung, rechtliche Unsicherheiten und technische Probleme (Viitanen et al. 2011).

Diese Beobachtung der fehlenden Durchdringung des Marktes beschrieb Heeks mit seinem Modell des „design-reality-gap“, das die „mismatches“ zwischen bestehenden Realitäten und Designkonzeptionen nachzeichnet (Heeks 2006, S. 128). Um diesen „gap“ überwinden zu können und zukünftig keine ,asocial and acontextual products" (May et al. 2003, S. 699) zu generieren, bedürfe es $u$. a. nach Martikainen et al. (2012, S. 99) einer stärkeren Einbindung von anderen Akteuren in den Technikentwicklungsprozess, insbesondere der zukünftigen Nutzer: „Several researchers have emphasized the need for [...] involving end-users in development activities". Die damit verbundene Hoffnung ist, dass Technologien entwickelt werden, die auch von potenziellen Nutzern akzeptiert werden (Blythe et al. 2005, S. 687; Schlick et al. 2010, S. 949ff.; Glende et al. 2009, S. 70ff.). Zur Einbindung zukünftiger Nutzer in Innovationsprozesse gibt es schon eine Vielzahl an erforschten und erprobten Ansätzen, wie bspw. ,user involvement" (Shah/Robinson 2006; Shah/Robinson 2007), „participatory design“ (Clemensen et al. 2007; Pilemalm/Timpka 2007), ,activity analysis“ (Häkkinnen/Korpela 2007), ,user- or human-centred design and development" (Johnson et al. 2005; Samaras/Horst 2005).

In der Technikfolgenabschätzung wird die unmittelbare Begleitung von technischen Entwicklungsprozessen konzeptionell im ,constructive Technology Assessment“ (cTA) umgesetzt (Rip et al. 1995). CTA, ursprünglich in den Niederlanden und Dänemark entwickelt, richtet 
dabei klassisch den Blick auf die möglichen gesellschaftlichen Folgen einer Technik, sucht aber gleichzeitig auch den Dialog zwischen und eine frühzeitige Interaktion mit neuen Akteuren, wie z. B. Konsumenten bzw. Nutzern und Produzenten. Durch ein sog. „,social mapping“ wird der gesellschaftliche Nutzungskontext der Technik erforscht, woraus sich dann relevante Akteure identifizieren lassen, die in den Technikentwicklungsprozess eingebunden werden sollten (ebd.). Die technische und die gesellschaftliche Entwicklung werden zusammen gedacht.

\section{Nutzereinbindung bei der Technikentwicklung zur Unterstützung von Menschen mit Demenz?}

Wenn bei der Erforschung des gesellschaftlichen Nutzungskontexts einer cTA allerdings Menschen mit Demenz als relevante „Techniknutzer" identifiziert werden, stellt das eine besondere methodische Herausforderung dar. Denn Menschen mit Demenz zeichnen sich durch sehr individuelle, oft tagesformabhängige Ausprägungen von Demenz aus, die es nötig machen, individuelle Krankheitsbilder und -verläufe mitzudenken. Dies führt zu dem Schluss, dass technische Lösungen stärker den komplexen Bedürfnissen und täglichen Anforderungen der Nutzer, eben den Menschen mit Demenz, gerecht werden müssen (Mollenkopf et al. 2005; Mulvenna/ Nugent 2010). Vielleicht ist diese methodische Schwierigkeit der Grund dafür, dass immer noch Bedürfnisse älterer Menschen (Glende et al. 2009), aber v. a. von Menschen mit Demenz, nur unzureichend bzw. gar nicht von Herstellern berücksichtigt werden. ${ }^{6}$ Ein Grund hierfür kann sein, dass ein empathisches Hineinversetzen durch zumeist junge Produktentwickler in ältere Nutzer schwierig erscheint bzw. sogar nicht möglich ist (Friesdorf/Heine 2007; Elsbernd et al. 2014). Hinzu kommt, dass die Heterogenität und Vielfalt von Nutzerbedürfnissen (Steger 1999; Cooper 2002) zu ausgeprägter Komplexität von Innovationsprozessen führen: „Technologieentwicklungen und Technologieerprobungen [werden] der Differenziertheit der Kunden und Nutzer methodisch nicht gerecht." (Liesenfeld/ Loss 2012, S. 270) „Typische Nutzungssitua- tionen sind in ihrer Komplexität nur schwer in konkrete Anforderungen umsetzbar und bleiben in einer flüchtigen Betrachtung zumeist unterbelichtet" (Compagna et al. 2010, S. 1).

Gleichzeitig wird aber gerade für die Entwicklung von Unterstützungssystemen für Menschen mit Demenz der Einblick in die Routinen des Pflegehandelns und die Lebensumstände von Menschen mit Demenz im Heim und im privaten Umfeld als zwingend notwendig erachtet (Bagnall 2006; Nykänen/Karimaa 2006): ,Die unmittelbare Beteiligung der Nutzer/innen ist unverzichtbar. $\mathrm{Zu}$ gewährleisten ist dabei, dass auch der Anwendungskontext der Technik einbezogen wird, also zum Beispiel der Lebensalltag, die täglichen Routinen, die familiären und privaten Beziehungen, der Sozialraum. Erst wenn technische Entwicklungen auch hier Bodenhaftung finden, können sie zu Dienstleistungsinnovationen beitragen.“(Liesenfeld/Loss 2012, S. 280)

Ein möglichst umfassendes Verständnis der Technikentwickler und Forscher des räumlichen, sozialen und funktionalen Kontextes, d. h. der komplexen Lebenslagen (Elsbernd et al. 2014), in denen die Technologie zukünftig genutzt werden soll, ist folglich unabdingbar. Dieses Verständnis umfasst auch die Relationen zwischen den verschiedenen Akteursgruppen im Handlungskontext sowie die normativen Strukturen, die dem Pflegekontext zugrunde liegen (May et al. 2003, S. 708).

\section{Bedarfsorientierte Vorgehensweise}

Diesem Gedanken soll in der hier skizzierten bedarfsorientierten TA nachgegangen werden. Diese nimmt das Pflegearrangement ${ }^{7}$ um den Mensch mit Demenz in den Blick, das aus dem Mensch mit Demenz, seinen Angehörigen, den professionell Pflegenden, den Ehrenamtlichen usw. besteht (Krings et al. 2014). Nun könnte man nach der oben beschriebenen Vorgehensweise des cTA alle diese Akteure in den Technikentwicklungsprozess einbinden und darauf vertrauen, dass sich dadurch die ursprüngliche Technikidee so entwickeln lässt, dass ihr Einsatz allen im Pflegearrangement akzeptabel und vielleicht sogar wünschenswert erscheint. Die hier vorgeschlagene Vorgehensweise setzt diesem 
Technology-push-Ansatz, denn es wird ja von einer konkreten technischen Lösung ausgegangen, eine Orientierung an den konkreten Bedarfslagen im Pflegearrangement entgegen (Demand-pull). Das heißt, statt mit einer Technikidee, die man unter Hinzuziehen möglichst vieler Akteure aus dem Pflegearrangement - also dem soziotechnischen Kontext - im Sinne eines cTA weiterentwickelt, wird mit einer Bedarfsanalyse im Pflegearrangement begonnen, die das Ziel hat, eine für das Pflegehandeln wünschenswerte mögliche technische Unterstützung zu identifizieren. Erst im Anschluss an dieses Modul der Bedarfsanalyse schließt sich eine klassische cTA an. Kurz: Der Bedarf an einer technischen Unterstützung wird erhoben und dann versucht, diese Unterstützung technisch mit einer zyklischen Einbindung von Nutzern zu entwickeln.

Das Ziel der Vorgehensweisen ist jeweils gleich: Es soll eine Technik entwickelt werden, die einen Bedarf an Unterstützung im Pflegearrangement so befriedigt, dass alle in diesem Arrangement die technische Unterstützung akzeptabel finden. Während aber im TechnologyPush möglicherweise viele Technologien analysiert werden müssen, bis eine dieser Technologien dieses Ziel erreicht und damit den Markt erfolgreich durchringen kann, wird beim Demand-pull die Chance, eine wünschenswerte Technikentwicklung zu realisieren von vornherein erhöht. Entscheidend bei diesem Ansatz ist, dass zunächst offen bleibt, welche Art Technik entwickelt werden soll. So wird letztendlich erst nach einer systematischen Bedarfserhebung eine Technologie vorgeschlagen, von der man als eine Art „Vertrauensvorschuss“ begründet annehmen kann, dass sie den identifizierten Bedarf befriedigen kann. Dabei orientiert sich die methodische Vorgehensweise der Bedarfserhebung an der empirischen qualitativen Sozialforschung. Möglichst alle Akteure im Pflegearrangement sollen zu ihrer Bedarfslage befragt werden.

\section{Bedarfserhebung: Fallbeispiel Menschen mit Demenz im Pflegeheim}

Zur Veranschaulichung der vorgeschlagenen Vorgehensweise der Nutzereinbindung in eine Bedarfsanalyse vor dem Beginn eines Technik- entwicklungsprojekts wird hier ein Handlungskontext vorgestellt, der in einem konkreten Forschungsprojekt „Movemenz-Mobiles, selbstbestimmtes Leben vom Menschen mit Demenz im Quartier" näher untersucht wurde.

Die Bedarfserhebung wurde im Feld der „stationären Pflege“ durchgeführt. Dabei wurde auf eine Methode der sozialwissenschaftlichen Feldforschung der sog. Teilnehmenden Beobachtung (nach z. B. Lüders 2003) zurückgegriffen, wobei eine Beobachtungsdauer von zwei Wochen realisiert werden konnte. In dieser Phase waren vier bis fünf Projektteam-Mitglieder im Pflegeheim als zurückhaltende, stille Beobachter von morgens bis abends vor Ort und beobachteten alle Akteure des Pflegearrangements. Diese Beobachtungsphase war eingebunden in ein methodisches Gesamtkonzept der sozialwissenschaftlich begründeten Grounded Theory, die - neben dem methodisch geleiteten Beobachten - vielseitige Formen der Datenerhebung miteinbezog. Nach dem Ansatz „All is Data“8 (Glaser 2007) wurden in Protokollen die Beobachtungen zu individuellen Akteuren und ihren Aktivitäten, Abläufen im Heim, sowie sozialen Rahmungen und Interaktionsordnungen, aber auch Hierarchien und Rollendifferenzierungen erfasst. Diese wurden anschließend $\mathrm{zu}$ Themenclustern verdichtet und hieraus Hypothesen abgeleitet. Die Erkenntnisse, die in dieser Phase gewonnen wurden, dienten der inhaltlichen Vorbereitung von Einzel- und Gruppeninterviews zur Reflexion der beobachteten Bedarfslagen. So wurden sowohl mit den Menschen mit Demenz sowie mit der Heimdirektion und der Pflegeleitung Einzelinterviews geführt. $^{9}$ In der Rollenverteilung eines Heims können die Hausdirektion und die Pflegedienstleitung aus ihrer Sicht möglicherweise andere Bedarfe äußern als beispielsweise die Pflegekräfte. Im Gegensatz dazu wurden die professionell Pflegenden, die Ehrenamtlichen und die Angehörigen der Menschen mit Demenz in je einer Gruppendiskussion im Stile einer Fokusgruppe (Stewart/Shamdasani 1990, S. 7; Flick 2010, S. 222ff.) befragt. Die leitfadengestützten Gruppeninterviews haben methodisch dahingehend einen Vorteil gegenüber Einzelinterviews mit allen Gruppenteilnehmern, dass vorgebrachte Argumente in der Gruppe bewertet 
werden. Aus dieser Diskussion kann man sowohl Argumentationszusammenhänge ableiten als auch Aufschlüsse zu einer relativen Bewertung von Argumenten erhalten. Ebenso bietet die Gruppensituation und die damit einhergehende Interaktion und Kommunikation der Teilnehmer miteinander den Vorteil, dass sich die Teilnehmer mit ihren Aussagen gegenseitig inspirieren und Themen auf diese Weise sehr viel umfassender, vielfältiger und zum Teil kreativer behandelt werden können als in Einzelinterviews. Im Leitfaden der Gruppen- und Einzelinterviews wurde nach einer einführenden Selbstbeschreibung durch die Interviewpartner jeweils zunächst nach allgemeinem Unterstützungsbedarf im Pflegehandeln gefragt, bevor konkreter ein möglicher Bedarf an technischer Unterstützung aus der individuellen und beruflichen Perspektive heraus diskutiert wurde. Darüber hinaus wurden die, aus den teilnehmenden Beobachtungen im Feld abgeleiteten Hypothesen zu Bedarfen erörtert ${ }^{10}$.

Eine methodische Herausforderung dieses Ansatzes bestand und besteht darin, dass die Bedarfe im Pflegearrangement ,nicht-technisch“ beschrieben werden. Sie beziehen sich auf das heute praktizierte Pflegehandeln. Auch wenn in den Einzel- und Gruppeninterviews jeweils gegen Ende gefragt wurde, ob man sich eine technische Unterstützung vorstellen könne, so wurden zwar einige Vorschläge zu Protokoll gegeben, aber es wurde ebenfalls die Schwierigkeit offensichtlich, pflegerische Bedarfe in mögliche technische Assistenz zu transformieren. Eine solche Transformationsleistung $\mathrm{zu}$ vollbringen, ohne eine fundierte technische Ausbildung zu haben, ist nicht einfach. Im Projekt „Movemenz“ wurde dieser Herausforderung durch das Einbinden von Technikentwicklern im weiteren Prozess begegnet. Diesen wurden die von den Pflegekräften, Ehrenamtlichen und Angehörigen nicht-technisch formulierten Bedarfe erläutert. Dann wurden sie gebeten, mögliche technische Lösungen für diese Bedarfslagen zu entwickeln. Zu diesen technischen Vorschlägen werden dann wieder die Akteure im Pflegearrangement befragt, ob damit die von ihnen geäußerten Bedarfe gedeckt werden könnten, um anschließend dieses Feedback erneut den Technikentwicklern zu spiegeln. Durch diese Iteration kann schließlich eine tech- nische Lösung entstehen, die zu einer konkreten Bedarfssituation im Pflegehandeln passt oder auch mehrere Bedarfe gleichzeitig adressiert.

Die aus dieser Bedarfsanalyse resultierenden technischen Assistenzsysteme könnten dann der Anfang eines „klassischen“ Technikentwicklungsprojektes werden, der seinerseits durch eine cTA begleitet werden sollte. In dem hier zugrunde liegenden Fallbeispiel mündet das Vorprojekt unmittelbar in diese cTA eines anschließenden Technikentwicklungsprojektes ein, um die gewonnen Erkenntnisse (s. unten) entsprechend einfließen zu lassen.

\section{Diskussion}

Das Projekt „Movemenz“ ist noch nicht abgeschlossen, daher können an dieser Stelle nur erste Erkenntnisse im Sinne von Zwischenergebnissen aus dem Arbeitsprozess heraus formuliert werden. Diese beziehen sich auf die hier vorgestellte Bedarfsanalyse, die zeitlich vor einer konkreten Technikentwicklung durchgeführt wird.

Mit Blick auf die Menschen mit Demenz, die man typischerweise als „Nutzer" einer assistiven Technik ansehen würde, bestätigt die Bedarfserhebung im untersuchten Fall, dass diese - wie oben bereits herausgestellt - nur bedingt als Interviewpartner einbezogen werden können. Aufgrund der kognitiven Einschränkungen und fehlenden Reflexionsmöglichkeiten der Menschen mit Demenz bleibt der Ertrag aus den Interviews begrenzt. Dies wurde verstärkt durch die Tatsache, dass sich im Untersuchungsfeld sehr individuelle, auf den Grad der Demenz und die jeweilige konkrete Verfasstheit bezogene Ausprägungen von Demenz zeigten. Hinzu kam, dass in dem ausgesuchten Pflegegeheim sehr alte Bewohner zwischen 84 und 96 Jahre leben und sich diese bis auf wenige Ausnahmen im späteren Stadium der Demenz befinden. In dem hier beschriebenen Fall wurden sechs Einzelinterviews mit Menschen mit Demenz durchgeführt. Diese Limitierung der Interviewpartner begründet sich darin, dass ein Dialog mit weiteren demenziell erkrankten Menschen aufgrund des Demenzverlaufs nach Einschätzung von Hausdirektion und Pflegedienstleitung nicht möglich sein würde. Wie in allen anderen Einzel- und Grup- 
peninterviews wurde auch bei den Menschen mit Demenz zunächst allgemein nach Bedarfen gefragt, die sie in ihrem Leben im Heim wahrnehmen. Sowohl ganz allgemein als auch in Bezug auf technisch adressierbare Bedarfe gab es keine konkreten Hinweise. Hier bestätigte sich die bereits vom Pflegepersonal vorab geäußerte Vermutung, dass es auch diesen sechs Menschen mit Demenz schon sehr schwer fällt bzw. nicht mehr möglich ist, ein hypothetisches Denken in Bedarfen und damit möglicher Weise verbundenen „Unterstützungsszenarien“ zu realisieren. In Bezug auf eine mögliche technische Unterstützung gab es unterschiedliche Antworten, die ihrerseits sehr allgemein blieben. Zwei Interviewpartnern war es nicht möglich, sich eine technische Unterstützung überhaupt vorzustellen. Ein weiterer Interviewpartner äußerte, dass er keine Unterstützung durch Technik bräuchte. Die restlichen Interviewten konnten generell eine technische Unterstützung in Betracht ziehen und reagierten zwar zurückhaltend aber dennoch positiv auf vorgeschlagene Technologien, die sie z. B. bei Spaziergängen unterstützen und ihnen ein ,Zurückfinden" erleichtern könnten.

Den anderen Akteuren im Pflegearrangement erscheint die Unterstützung von Menschen mit Demenz durch eine technische Lösung mit Einschränkungen durchaus plausibel. Allerdings wurde auch von diesen Akteuren auf Schwierigkeiten hingewiesen. So merkte in der Studie von Hirsch eine Pflegeberaterin an:
„Man braucht viel Zeit, um sich auf Menschen mit Demenz einlassen zu können, man kann Menschen [mit Demenz] nicht ans System an- passen [...], das geht bei 'nem Demenzkran- ken nicht, das System muss sich an den Betrof- fenen anpassen. “4

Auch allgemeiner wurde eine technische Unterstützung für die Menschen mit Demenz kritisch gesehen:
„Menschen mit Demenz zeigen gegenüber Technik Abwehrverhalten, z. B. muss ein Lifter [mobile Pflege- und Transferhilfe] immer wie- der in kleinen Schritten erklärt werden, damit sie keine Angst mehr davor haben." (Aussage einer Pflegekraft in Ausbildung) ${ }^{4}$

Das bestätigt die Vermutung, dass viele Menschen mit Demenz im mittleren bis späterem
Stadium nur in eingeschränkter Weise unmittelbar (und allein) Nutzer einer assistiven Technik sein können. Dies kann aufgrund der sehr starken individuellen Ausprägung von Demenz natürlich nicht allgemein gültig festgestellt werden, da ein Mensch mit Demenz im mittleren Stadium diese noch nutzen kann, während ein anderer es nicht (mehr) kann.

Durch die Betrachtung des gesamten Pflegearrangements bei der hier verfolgten bedarfsorientierten Vorgehensweise rücken aber auch andere mögliche Techniknutzer in den Fokus. Diese Erkenntnis kann als ein Resultat der bedarfsorientierten Vorgehensweise festgehalten werden: Sie ist demnach nicht nur offen bezüglich einer zu beschreibenden Technik, sondern auch offen bezüglich der „Nutzer“ dieser Technik. Auch wenn das Pflegearrangement eingerichtet wird, um einen Mensch mit Demenz im Pflegeheim bestmöglich zu unterstützen, so muss der Mensch mit Demenz nicht selbst der Nutzer der Technik sein. Er kann auch einen Nutzen dadurch haben, dass ein anderer Akteur im Arrangement eine technische Unterstützung einsetzt, um seine Aufgabe im Pflegearrangement „besser" erfüllen zu können:

„[...] Das wäre dann eher so etwas Indirektes, dass wir eine Technik haben, [...], [die] uns andere Arbeiten abnimmt, dass wir für [...] etwas mehr Zeit haben, auf so einem indirekten Weg vielleicht auch.“ (Aussagen einer Pflegekraft im Gruppeninterview $)^{4}$

Es lässt sich also festhalten, dass sich mithilfe der bedarfsorientierten Vorgehensweise technische Lösungskonzepte identifizieren lassen, die von einzelnen Akteuren im Pflegearrangement genutzt werden oder auch zwischen verschiedenen Akteuren - wie Kommunikationstechnologien solange sie nur als Lösung für einen Bedarf oder mehrere Bedarfe allen im Arrangement akzeptabel erscheint ${ }^{11}$. Weiterführend ist durch die bisherigen Ergebnisse belegt, dass mit der bedarfsorientierten Vorgehensweise auch viele Bedarfe herausgearbeitet werden, die nicht in eine mögliche technische Lösung überführt werden können. Dazu gehören auch und gerade Probleme im Pflegealltag, die sich aus den Auswirkungen von gesundheitspolitischen Vorgaben ergeben. Kostendruck und Betreuungsverhältnisse, die ei- 
nen verantwortlichen Umgang mit den speziellen Bedürfnissen von Menschen mit Demenz kaum zulassen, seien hier stellvertretend genannt.

Im Projekt „Movemenz“ wird ein Aufgreifen dieser - nicht durch technische Lösung adressierbaren Bedarfe - durch einen begleitenden „Runden Tisch“ sichergestellt. Dieser setzt sich aus wissenschaftlichen ExpertInnen der technischen, ethischen, rechtlichen, pflege- und sozialwissenschaftlichen Disziplinen zusammen. In diesem Gremium werden aus der empirischen Bedarfsforschung aber gleichermaßen aus den unterschiedlichen wissenschaftlichen Disziplinen Handlungsoptionen für politische Entscheider und die allgemeine Öffentlichkeit entwickelt. Für die Diskussion mit den Akteuren im Pflegearrangement ist dieser begleitende Diskussionsprozess, der die Berücksichtigung der Bedürfnisse sicherstellt, die sich nicht durch technische Assistenzsysteme befriedigen lassen, in doppelter Hinsicht wichtig. Zum einen lässt sich in den Texten des Runden Tisches nachvollziehen, dass auch diese nicht-technischen Bedarfe in der weiteren Diskussion berücksichtigt werden. Zum anderen hilft dieser Ansatz, Vertrauen zu schaffen, sich auf die technischen Optionen „einzulassen“. Denn natürlich bestehen auch Befürchtungen, dass durch Technik u. a. Ersetzungen von menschlichen Fähigkeiten und Personen stattfinden. Beispielsweise wären dann „,ie [Physiotherapeuten] in einem Pflegeheim zum größten Teil arbeitslos" wie eine Pflegekraft im Gruppeninterview zu bedenken gab. Darüber hinaus ist der Runde Tisch als Instrument vorgesehen, den Transfer der Diskussionsergebnisse aus dem Vorprojekt in das anschließende Technikentwicklungsprojekt sicherzustellen. Damit würde auch die cTA in diesem Projekt von den Erkenntnissen aus dem Vorprojekt profitieren.

Zusammenfassend lässt sich konstatieren, dass mit der bedarfsorientierten Technikentwicklung sehr wohl die Hoffnung verbunden sein kann, dass Technologien entwickelt werden, von denen man dann davon ausgehen kann, dass sie in bestehenden Pflegearrangements für akzeptabel erachtet werden. Damit steigt die Chance, dass Technik innoviert wird, die den Markt „Pflege" durchdringt und im Pflegealltag nachhaltig Verwendung findet. Vor allem, wenn während der Technikentwicklungsphase die Akteure des Pflegearrangements in einem cTA eingebunden bleiben, so dass diese Akzeptabilität auch bei der Technikentwicklung erhalten bleibt. Das kann ebenso die ersten Testphasen der neu entwickelten Technik im Pflegeheim umfassen. Diesen vorgeschlagenen Ansatz halten die professionell Pflegenden jedenfalls für verfolgenswert:

\begin{abstract}
„Aber bisher war ja Technik immer, man hat ein Gerät erfunden und geschaut, wer könnte es nutzen. Das fällt mir oft auf, dass Technik immer entwickelt wird und dann geschaut wird, wo kann man es einsetzen, statt - aber das machen Sie ja in dem Falle Gott sei Dank andersherum - erst zu schauen, was sind die Bedürfnisse und wie kann man so etwas individualisieren." (Aussage der Hausdirektion im Einzelinterview) ${ }^{4}$
\end{abstract}

\section{Danksagung}

Die Autoren danken den Angestellten und Bewohnern des untersuchten Pflegeheims, welche diese Arbeit durch ihr Engagement erst möglich gemacht haben, und der Evangelischen Heimstiftung (EHS) für den uneingeschränkten $\mathrm{Zu}-$ gang zum Pflegeheim und die Unterstützung der Forschung. Wir sind ebenso dankbar für die Beiträge von Bettina-Johanna Krings, Johannes Hirsch, Silvia Woll und Marcel Krüger im Projekt Movemenz „Mobiles, selbstbestimmtes Leben vom Menschen mit Demenz im Quartier", in dem auch die hier vorgestellten Ergebnisse erarbeitet wurden. Gefördert wird das Projekt vom Bundesministerium für Bildung und Forschung (BMBF).

\section{Anmerkungen}

1) http://www.bmg.bund.de/themen/pflege/pflegekraefte/pflegefachkraeftemangel.html

2) „Assistive technology is any device or system that allows an individual to perform a task that they would otherwise be unable to do, or increases the ease and safety with which the task can be performed" (Royal Commission on Long Term Care 1999, S. 382; zit. n. Siota/Simpson 2008). Die Produkte reichen hierbei von „low-tech“ Gegenständen, wie Stöcke und Handläufe, über 
mechanische und elektrische Geräte, z. B. manuell und elektrisch angetriebenen Rollstühle, bis hin zu elektronischen sowie Informations- und Kommunikationstechnologie (IKT) Systemen. In diesem Beitrag wird der Begriff der assistiven Technologie enger gerahmt: Technologien, die heute zur Standardausstattung eines Heims gehören und von den Bewohnerinnen und Bewohner tagtäglich genutzt werden, wie z. B. die Pflegedokumentation, Rollatoren und Rollstühle sowie Gehbrücken, sind im verwendeten Technologiebegriff exkludiert.

3) http://www.mtidw.de/service-und-termine/publikationen/besser-leben-im-alter-durch-technik

4) Zitat aus den Transkripten der von Hirsch durchgeführten Einzelinterviews, siehe auch Hirsch 2015.

5) https://www.wegweiseralterundtechnik.de

6) Unzureichend in dem Sinne, dass in den meisten Fällen Technologien entwickelt werden, von denen lediglich angenommen wird, dass sie einen Bedarf adressieren.

7) Den Begriff des Pflegearrangement haben Blinkert/Klie (2004) geprägt und beziehen sich hierbei auf ein Konzept, das Pflegebedarf und Pflegetätigkeit in ein spezifisches Verhältnis setzt. So kann man auf der Basis des Konzepts analysieren, wie und in welchem Ausmaß verschiedene Akteure an der Versorgung pflegebedürftiger Menschen beteiligt sind. Hierbei werden Fragen nach der Art der Pflege und nach dem Verhältnis professioneller und informeller Pflege genauso gestellt, wie nach der „Vermischung“ häuslicher und stationärer Pflege.

8) Der Ansatz „All is data“ ist auch bekannt unter „Glaser dictum“: „It means that exactly what is going on in the research scene is data, whatever the source, whether interview, observations, documents. It is not just what is being, how it is being and the conditions of its being told, but all the data surrounding what is being told. It means what is going on has to be figured out exactly what it is for conceptualization, NOT [Hervorheb. vom Autor] description. Data is always as good as far as it goes, and there is always more to keep correcting the categories with more relevant properties." (Glaser 2007, S. 1)

9) Einzelinterviews, da zum einen die Menschen mit Demenz aufgrund ihrer körperlichen und geistigen Verfasstheit nicht in der Lage waren, an Gruppeninterviews in adäquater Weise teilzunehmen. Und zum anderen, um für die anderen Interviewpartner, wie der Hausdirektion und der Pflegeleitung, einen abgeschirmten Raum zu kreieren und dabei ihre besondere Perspektive auf Aktivitäten im Pflegealltag einzufangen.

10) Detaillierte Ausführungen zum Projekt, seinem Ablauf und ersten Ergebnissen sind in Weinberger et al. (2014), Weinberger et al. (2015) und Decker/Weinberger (2015) sowie auf der Projekthomepage zu finden.

11) Mit der Akzeptabilität kann hier auch eine $\mathrm{Zu}-$ mutung für Akteure im Pflegearrangement verbunden sein, wenn diese Zumutung normativ akzeptabel ist, um einen Bedarf im Arrangement zu befriedigen. Beispielsweise könnte es für Angehörige zumutbar sein, sich erst über ein Tablet über den Aufenthaltsort des Menschen mit Demenz informieren zu müssen. Damit ist auch der Unterschied zwischen einer faktischen Akzeptanz und der Akzeptabilität gekennzeichnet. Eine solche Zumutung wird möglicherweise nicht akzeptiert, sondern lediglich in Kauf genommen, um im Pflegearrangement einen Vorteil zu erlangen. Idealerweise müssen sich alle Akteure im Pflegearrangement an diesem Diskurs beteiligen. Im vorliegenden Fall war dies den Menschen mit Demenz nicht mehr möglich, so dass ihre Interessen durch die Angehörigen und die professionell und ehrenamtlich Pflegenden vertreten werden mussten.

\section{Literatur}

Bagnall, P.; Onditi, V.; Rouncefield, M. et al., 2006: Older People, Technology and Design - A Socio-technical Approach. In: Gerontechnology 5/1 (2006), S. 46-50

Blinkert, B.; Klie, T., 2004: Solidarität in Gefahr: Pflegebereitschaft und Pflegebedarfsentwicklung im demografischen und sozialen Wandel; die „Kasseler Studie". Hannover

Blythe, M.A.; Monk, A.F.; Doughty, K., 2005: Socially Dependable Design - The Challenge of Ageing Populations for HCI. In: Interacting with Computers 17 (2005), S. 672-689

Clemensen, J.; Larsen, S.B.; Kyng, M. et al., 2007: Participatory Design in Health Sciences: Using Cooperative Experimental Methods in Developing Health Services and Computer Technology, In: Qualitative Health Research 17/1 (2007), S. 122-130

Compagna, D.; Derpmann, S.; Graf, B. et al., 2010: Anwenderorientierte Technikentwicklung im PflegeBereich: Instrumente für den Wissenstransfer zur partizipativen Gestaltung von Mikrosystemtechnik. Dritter Deutscher AAL-Kongress 26.-27. Januar 2010, Berlin 
Cooper, R.G., 2002: Top oder Flop in der Produktentwicklung. Weinheim

Decker, M.; Weinberger, N., 2015: Was sollen wir wollen - Möglichkeiten und Grenzen der bedarfsorientierten Technikentwicklung. In: Weidner, R.; Redlich, T. (Hg): Technische Unterstützungssysteme, die die Menschen wirklich wollen. Berlin/Heidelberg (im Druck)

Elsbernd, A.; Lehmeyer, S.; Schilling, U., 2014: So leben ältere und pflegebedürftige Menschen in Deutschland. Lebenslagen und Technikentwicklung. Lage

Fachinger, U.; Henke, K.-D. (Hg.), 2010: Der private Haushalt als Gesundheitsstandort. Theoretische und empirische Analysen. Baden-Baden

Ferri, C.; Prince, M.; Brayne, C. et al., 2005: Global Prevalence of Dementia: A Delphi Consensus Study. In: Lancet 366 (2005), S. 2112-2117

Flick, U., 2010: Qualitative Sozialforschung. Eine Einführung. Berlin

Friesdorf, W.; Heine, A., 2007: Sentha - seniorengerechte Technik im häuslichen Alltag. Berlin

Geyer, J., 2015: Einkommen und Vermögen der Pflegehaushalte in Deutschland, In: DIW-Wochenbericht, 82/14-15 (2015), S. 323-328

Glaser, B.G., 2007: All is Data. In: The Grounded Theory Review 6/2 (2007), S. 1-22

Glende, S.; Nedopil, C.; Podtschaske, B. et al., 2011: Nutzerabhängige Innovationsbarrieren im Bereich altersgerechter Assistenzsysteme. 1. Studie im Rahmen der AAL-Begleitforschung des Bundesministeriums für Bildung und Forschung. Abschlussbericht

Glende, S.; Podtschaske, B.; Friesdorf, W., 2009: Senior User Integration: Ein ganzheitliches Konzept zur Kooperation von Herstellern und älteren Nutzern während der Produktentwicklung. Conference: Ambient Assisted Living - AAL - 2. Deutscher AAL-Kongress mit Ausstellung/Technologien - Anwendungen - Management. 27.-28. Januar 2009, Berlin

Häkkinen, H.; Korpela, M., 2007: A Participatory Assessment of IS Integration Needs in Maternity Clinics Using Activity Theory, In: International Journal of Medical Informatics 76 (2007), S. 843-849

Haux, R.; Hein, A.; Kolb, G. et al., 2014: Information and Communication Technologies for Promoting and Sustaining Quality of Life, Health and Self-sufficiency in Ageing Societies - Outcomes of the Lower Saxony Research Network Design of Environments for Ageing (GAL). In: Informatics for Health and Social Care 39/3-4 (2014), S. 166-187

Heeks, R., 2006: Health Information Systems: Failure Success and Improvisation. In: International Journal of Medical Informatics 75 (2006), S. 125-137
Hersh, W.R.; Helfand, M.; Wallace, J. et al., 2001: Clinical Outcomes Resulting from Telemedicine Interventions: A Systematic Review. In: BMC Medical Informatics and Decision Making 1/5 (2001)

Hirsch, J., 2015: Technik, die gewollt ist - Ein Vergleich von Technikentwicklungsansätzen zur Unterstützung von Menschen mit Demenz. Diplomarbeit am Institut für Anthropomatik und Robotik und am Institut für Technikfolgenabschätzung und Systemanalyse

Hülsken-Giesler, M., 2010: Technikkompetenzen in der Pflege - Anforderungen im Kontext der Etablierung neuer Technologien in der Gesundheitsversorgung, In: Pflege \& Gesellschaft 15/4 (2010), S. 330-352

Johnson, C.M.; Johnson, T.R.; Zhang, J., 2005: A User-centered Framework for Redesign Healthcare Interfaces, In: Journal of Biomedical Informatics 38 (2005), S. 75-87

Krings, B.-J.; Böhle, K.; Decker, M. et al., 2014: ITAMonitoring „Serviceroboter in Pflegearrangements“. In: Decker, M.; Fleischer, T.; Schippl, J. et al. (Hg.): Zukünftig Themen der Innovations- und Technikanalyse. Lessons Learned und ausgewählte Ergebnisse. KIT Scientific Reports. Karlsruhe, S. 63-122

Liesenfeld, J.; Loss, K., 2012: Innovative AAL- und E-Health-Dienstleistungen: Zusammenhänge zwischen technologischen Entwicklungen, Geschäftsmodellen und Governance. In: Gersch, M.; Liesenfeld, J. (Hg.): AAL- und E-Health-Geschäftsmodelle. Wiesbaden, S. 265-285

Lüders, C., 2003: Teilnehmende Beobachtung. In: Bohnsack, R.; Marotzki, W.G.; Meuser, M. (Hg.): Hauptbegriffe Qualitativer Sozialforschung. Opladen, S. 151-153

Martikainen, S.; Vitanen, J.; Korpela, M. et al., 2012: Physicians' Experiences of Participation in Healthcare IT Development in Finland: Willing But Not Able. In: International Journal of Medical Informatics 81 (2012), S. 99-113

May, C.; Mort, M.; Williams, T. et al., 2003: Health Technology Assessment in its Local Contexts: Studies of Telehealthcare. In: Social Science and Medicine 57 (2003), S. 697-710

Mollenkopf, H.; Schakib-Ekbatan, K.; Oswald, F. et al., 2005: Technische Unterstützung zur Erhaltung von Lebensqualität im Wohnbereich bei Demenz. Ergebnisse einer Literatur-Recherche. Forschungsberichte aus dem Deutsches Zentrum für Alternsforschung an der Universität Heidelberg, Abteilung für Soziale und Ökologische Gerontologie, Nr. 19

Mulvenna, M.D.; Nugent, C.D. (Hg.), 2010: Supporting People with Dementia Using Pervasive Health Technologies. New York 
Mulvenna, M.D.; Nugent, C.D.; Moelaert, F. et al., 2010: Supporting People with Dementia Using Pervasive Healthcare Technologies. In: Mulvenna, M.D.; Nugent, C.D. (Hg.): Supporting People with Dementia Using Pervasive Health Technologies. London, S. 3-19

Nykänen, P.; Karimaa, E., 2006: Success and Failure Factors in the Regional Health Information System Design Process - Result from a Constructive Evaluation Study. In: Methods of Information in Medicine 45/1 (2006), S. 85-89

Pilemalm, S.; Timpka, T., 2007: Third Generation Participatory Design in Health Informatics - Making User Participation Applicable to Large-scale Information System Projects, In: Journal of Biomedical Informatics 41 (2007), S. 327-339

Prince, M.; Albanese, E.; Guerchet, M. et al., 2014: Dementia and Risk Reduction. An Analysis of protective and modifiable factors. In: World Alzheimer Report 2014. Alzheimer's Disease International

Rachmann, A.; Maucher, I.; Schöler, B.; Hewing, M., 2011: Benutzerzentriertes Service Engineering am Beispiel einer Telemonitoring-Dienstleistung. In: Bieber, D.; Schwarz, K. (Hg.): Mit AAL-Dienstleistungen altern. Nutzerbedarfsanalysen im Kontext des Ambient Assisted Living, Saarbrücken, S. 197-218

Rip, A.; Misa, T.J.; Schot, J., 1995: Managing Technology in Society. London

Roine, R.; Ohinmaa, A.; Hailey, D., 2001: Assessing Telemedicine: A Systematic Review of the Literature. In: Canadian Medical Association Journal, 165 (2001), S. 765-771

Royal Commission on Long Term Care, 1999: With Respect to Old Age: Long Term Care - Rights and Responsibilities. TSO - The Stationery Office

Samaras, G.M.; Horst, R.L., 2005: A System Engineering Perspective on the Human-centred Design of Health Information Systems. In: Journal of Biomedical Informatics 38 (2005), S. 61-74

Schlick, C.M.; Bruder, R.; Luczak, H., 2010: Arbeitswissenschaft. Berlin

Shah, S.G.S; Robinson, I., 2006: User Involvement in Healthcare Technology Development and Assessment: Structured Literature Review. In: International Journal of Health Care Quality Assurance 19/6 (2006), S. 500-515

Shah, S.G.S.; Robinson, I., 2007: Benefits of and Barriers to Involving Users in Medical Device Technology Development and Evaluation, In: International Journal of Technology Assessment in Health Care 23/1 (2007), S. 131-137
Siota, R.; Simpson, C., 2008: Applying Telecare in Dementia: What Psychiatrists Need to Know. In: Advances in Psychiatric Treatment 14 (2008), S. 382-388 Steger, U., 1999: Facetten der Globalisierung. Berlin Stewart, D.W.; Shamdasani, P.N., 1990: Focus Groups: Theory and Practice. London

Viitanen, J.; Nieminen, M.; Hypponen, H. et al., 2011: Finnish Physicians' Experiences with Computer-supported Patient Information Exchange and Communication in Clinical work. In: International Journal of Electronic Healthcare 6/2-4 (2011), S. 153-173

Weinberger, N.; Decker, M.; Krings, B.-J., 2014: Pflege von Menschen mit Demenz - Bedarfsorientierte Technikgestaltung. In: Schultz, T.; Putze, F.; Kruse, A. (Hg.): Technische Unterstützung für Menschen mit Demenz, Symposium 30.9.-1.10.13. Karlsruhe

Weinberger, N.; Krings, B.-J.; Decker, M., 2015: Enabling a Mobile and Independent Way of Life for People With Dementia - Needs-oriented Technology Development. In: Dominguez-Rué, E.; Nierling, L. (Hg.): Ageing and Technology: Perspectives from the Social Sciences (im Druck)

Whitten, P.; Mair, F.; Haycox, A. et al., 2002: Systematic Review of Cost Effectiveness Studies of Telehealthcare Interventions. In: British Medical Journal 324 (2002), S. 1434-1437

\section{Kontakt}

Dipl.-Ing. Nora Weinberger

Institut für Technikfolgenabschätzung und

Systemanalyse (ITAS)

Karlsruher Institut für Technologie (KIT)

Karlstraße 11, 76133 Karlsruhe

Tel.: +49 721 608-23972

E-Mail: nora.weinberger@kit.edu 